\title{
Smokeless Tobacco Use among Pregnant Women in India: The Tale of Two Nationally Representative Surveys
}

\author{
Prashant Kumar Singh*, Pankhuri Jain, Nishikant Singh, Lucky Singh, \\ Shalini Singh
}

\begin{abstract}
The prevalence of smokeless tobacco (SLT) is pervasive in many Asian countries, including India. SLT use among pregnant women is markedly high, and is considered to be a global concern. Consequently, the associated health effects of SLT consumption on pregnant women and the foetus cause long-term adverse effects. Therefore, this article aims to understand the extent of deviation in SLT use estimates among Indian pregnant women based on two nationally representative surveys and its implications. Responses of 1,403 pregnant women were recorded in the Global Adult Tobacco Survey (GATS 2016-17), whereas 184,641 pregnant women were interviewed in the fourth round of the Demographic and Health Survey or the National Family Health Survey (DHS NFHS 2015-16). Considerable differences in SLT use patterns among pregnant women between the GATS-2 and the NFHS-4 was evident. Overall, the prevalence of SLT use among pregnant women was reported to be higher in GATS than NFHS and this pattern remains similar between age groups of 15 to 34. In the absence of reliable estimates of SLT use among pregnant women, its adverse implications for both reproductive, maternal and child health policies, tobacco control efforts and data quality issues need to be acknowledged and addressed in a targeted manner.
\end{abstract}

Keywords: Pregnant women- smokeless tobacco- maternal and child health- India

Asian Pac J Cancer Prev, 23 (2), 389-392

\section{Introduction}

Unlike smoking, the prevalence of smokeless tobacco is considerably higher among women in many Southeast Asian countries with 71 million female users in India alone(CDC Foundation et al., 2015). Smokeless tobacco or SLT is a tobacco product consumed without being burnt at the time of use, commonly consumed orally or nasally. These products can be placed in the mouth, cheek, or the lip and are sucked or chewed (Boffetta et al., 2008). They are often used for gargling, and also as dentifrice as a means for nicotine to be absorbed through the lining of the mouth (Bhisey, 2012). Evidence demonstrates that ubiquitous use of SLT has been not just been reported among adult males, but also among children, teenagers, women of reproductive age and immigrants of Southeast Asia wherever they have migrated and settled (Jatoi et al., 2009). Looking at the global pattern of SLT use among pregnant women, a study found Southeast Asia on the top of the chart with the highest rates of consumption. However, widespread use of SLT was also reported in other parts of the world. Hence, SLT use among pregnant women has been deemed as a global concern (Caleyachetty et al., 2014). The main aim of this study is to examine the extent of SLT use among pregnant women in India using two latest nationally representative surveys and discuss its implications on reproductive, maternal and child health programmes, tobacco control efforts and data quality issues within this vulnerable group.

\section{Materials and Methods}

\section{Data and Methods}

The Global Tobacco Surveillance System collects and maintains global datasets on use, prevalence and patterns of tobacco, including data on SLT use, across WHO regions through four surveys: Global Youth Tobacco Survey (GYTS); Global School Personnel Survey (GSPS); Global Health Professions Student Survey (GHPSS) and Global Adult Tobacco Survey (GATS) (Tata Institute of Social Sciences and Ministry of Health and Family Welfare Government of India, 2017). In India, data on SLT use and prevalence among pregnant women is collected by the GATS and the National Family Health Survey (NFHS), the India-specific version of the Demographic and Health Survey (DHS).

The GATS remains to be the foremost dataset on tobacco related data across many nations, including 
India. The first round of GATS was implemented in 2009-10. However, only the second round, implemented in 2016-17, collected information on pregnancy status in conjunction with SLT use (International Institute for Population Sciences (IIPS) and ICF, 2017). The NFHS, however, has been an ever-evolving survey, and covers aspects on maternal and child health making it the most reliable source of the status of various aspects of health information across the country. It was launched in the year 1991, the first round was conducted in 1992-93, succeeded by round 2 in the year 1998-99. However, it was only from the NFHS round 3 onwards, in 2005-06, that data on SLT use in conjunction with pregnancy status was collected. The latest round of the NFHS, was implemented in 201516 and covers 29 States and 7 Union Territories. The data in NFHS was obtained from 601,509 households in 28,521 Primary Sampling Units (PSUs) between January 20, 2015 and December 4, 2016 with the response rate of $97.6 \%$, with an individual response rate of $96.7 \%$. In GATS, data was obtained from 84,047 Households and 76,500 PSUs from August 2016 to February 2017 with a household response rate of $92.9 \%$ and individual-response rate of $96.0 \%$.

This study used data from the latest rounds of the GATS (2016-17) and the DHS-NFHS (2015-16) to estimate the prevalence of SLT use among pregnant women. Both the surveys utilise multi-stage sampling techniques and have collected self-reported information related to SLT use among pregnant women aged 15-49 years. Conducted across 30 Indian states and 2 Union Territories, GATS-2 survey included 1,403 pregnant women (ages 15-49) whereas the NFHS-4 included 184,641 pregnant women (ages 15-49). Further details related to sampling, stratification, response rate and questions of GATS and NFHS could be found elsewhere (International Institute for Population Sciences (IIPS) and ICF, 2017; Tata Institute of Social Sciences and Ministry of Health and Family Welfare Government of India, 2017).

\section{Results}

It was found that the national prevalence of any SLT use among pregnant women was 4\% in NFHS (2015-16). However, on the other hand, the second round of GATS (2016-17) suggests that the estimated prevalence of SLT use among pregnant women in India was $7.4 \%$, translating to a gross mismatch in the two most cited health information resources in the country (Figure 1). Further, when examining the age-wise patterns of SLT use prevalence among pregnant women, NFHS shows a rise with the increase in women's age. For instance, $3 \%$ of the pregnant women aged 20-24 were consuming SLT according to the NFHS, while the corresponding percentage was $6.3 \%$ and $9.5 \%$ among $30-34$ and 35-39 age groups respectively. In case of GATS, age wise pattern suggests increasing pattern of SLT use till 25-29 age groups and a subsequent decline thereafter. However, there is a conspicuous difference among the two surveys in the reported SLT use from ages 20 to 34 years. For instance, SLT use among pregnant women aged 25-29 was $24 \%$ as per the GATS, whereas the corresponding figure was $4 \%$ in case of NFHS - a gap of nearly five times.

\section{Discussion}

Our findings suggest that there is a substantial difference in the estimates of smokeless tobacco use among pregnant women as generated by the National Family Health Survey and the Global Adult Tobacco Survey. This finding is consistent with the extant literature as self-reporting methods lead to underestimating the true prevalence of tobacco use, especially among women as these surveys tend to evoke socially desirable responses and may lead to discrepancies in datasets (Roth et al., 2009; Shipton et al., 2009).

The substantial difference in SLT use among pregnant women between two large-scale nationally representative surveys has significant implications for both reproductive,

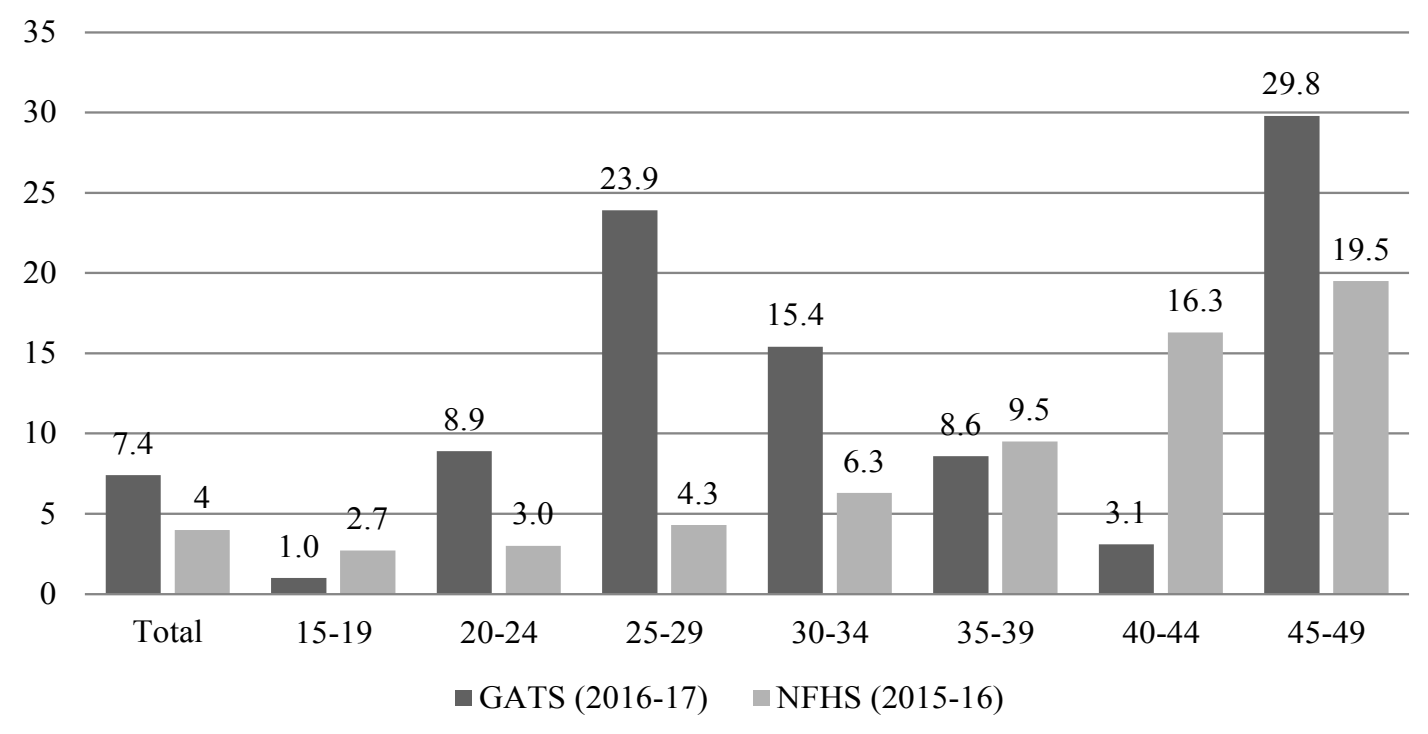

Figure 1. Percentage of Pregnant Women Reported Any form of Smokeless Tobacco Use, India. Source: generated by the author; Refs. 6 \& 7. GATS: Global Adult Tobacco Survey; NFHS: National Family and Health Survey 
maternal and child health policies and tobacco control efforts in India and other Southeast Asian countries with high prevalence of SLT use.

First, the current estimates of smokeless tobacco use reporting are not sufficient to understand and gauge the scope of the tobacco use burden among pregnant women. Since estimates of tobacco use guide the development of appropriate strategies for tobacco control and thus, if current tobacco uses remains undermined, adequate cessation support may not be available even in the presence of intention to quit.

Second, poor reproductive, maternal, new born and child health and nutrition (RMNCH\&N) outcomes remain to be a major public health concern in the country. For instance, nearly half of the pregnant women are anaemic as per the NFHS 2015-16 and it has only marginally declined from NFHS 2005-06 (58\%). Similarly, one out of five births that took place in India was less than 2.5 kilograms (low birth weight). Moreover, postpartum complications have increased over the period of time (Ashfaq et al., 2008). Existing studies have shown infertility, degenerative placental changes (Agrawal et al., 1983; Mughal et al., 2019), increased placental weight (Pratinidhi et al., 2010), pregnancy complications (Suliankatchi \& Sinha, 2016), pre-term delivery, low-birth weight, increased stillbirth risk (Rogers, 2008) and risk of cancers in the developing foetus (Yadav et al., 2020) as some of the adverse health effects of SLT use during pregnancy. In the absence of reliable data on SLT use among pregnant women various on-going programmes related to maternal and child health completely overlooks SLT use as a potential threat. Moreover, on-going RMNCH\&N programmes refrain to design any targeted interventions aimed towards the pregnant women who consume SLT due to such discrepancy in data.

Third, India has launched the National Tobacco Control Programme (NTCP) in 2007-2008, a commitment towards the provision and more effective implementation and enforcement of tobacco control measures under COTPA (Cigarettes and Other Tobacco Products (Prohibition of Advertisement and Regulation of Trade and Commerce, Production, Supply and Distribution Act, 2003) and WHO FCTC (World Health Organization Framework Convention on Tobacco Control) (Yadav et al., 2020). Main objectives of NTCP are (a) to increase awareness about existing tobacco control laws and the harmful effects of tobacco use; and (b) to facilitate effective implementation of tobacco control laws and policies. Furthermore, NTCP activities include expansion of tobacco cessation facilities, school-based tobacco control programmes, training and capacity building for teachers, health professionals and other stakeholders, and monitoring of tobacco control activities (National Tobacco Control Cell et al., 2015). It is possible that in the absence of accurate estimate of SLT among pregnant women, NTPC lacks clarity and this highly vulnerable section of the population may have been neglected. For instance, any report or guidelines pertaining to SLT use cessation strategies among the pregnant women in India could not be found.

Fourth, in a country where tobacco use revolves around gender norms and social taboos, possibility of under-reporting and socially desirable responses cannot be overlooked. Thus, it is quite likely that presence of any other household member or husband during the interview of a pregnant woman may significantly undermine the positive response. More research and discussion are required to devise tools that would be able to gather more reliable ways of collection of information from pregnant women on sensitive risk factors, like SLT use.

Fifth, more emphasis needs to be laid on migrant populations and should be included in GATS as they may be more susceptible to risky practices due to psychological stressors and low access to health resources and services (Borhade, 2011). Further, migrant pregnant women may be using regional variants of SLT, which have not been previously documented and hence left behind a sizeable number of populations from the current SLT estimates. Thus, emphasis on capturing migrant populations must be made for the future rounds of both surveys.

\section{Limitation}

This study utilises cross-sectional data on the prevalence of SLT use among pregnant women and thus causative relationships cannot be established. Further, the information on SLT use was self-reported; may be subject to socially desirable responses, reporting bias and is difficult to verify.

To conclude, SLT use among the pregnant women has been neglected for long and it is taking an unaccounted toll on various aspects of $\mathrm{RMNCH}$ outcomes in the country. Thus, strengthening data quality and developing reliable estimates must be the central focus to achieve both national as well as the global goals of improving health and promoting wellbeing for all.

\section{Author Contribution Statement}

\section{NOTE!!!}

The contributions of all authors must be described in the following manner: The authors confirm contribution to the paper as follows: study conception and design: $\mathrm{X}$. Author, Y. Author; data collection: Y. Author; analysis and interpretation of results: X. Author, Y. Author. Z. Author; draft manuscript preparation: Y. Author. Z. Author. All authors reviewed the results and approved the final version of the manuscript.

\section{Acknowledgments}

None.

\section{Data Availability Statement}

This study uses publicly available data, available from: https://www. dhsprogram.com/data/dataset/India Standard-DHS_2015.cfm?flag $=0$

\section{Competing interests}

None declared. 


\section{Ethics Approval}

The study was reviewed by the National Institute of Cancer Prevention and Research Institutional Ethics Committee and was exempted from full review as the study was based on an anonymous public use dataset with no identifiable information on the study participants.

\section{References}

Agrawal P, Chansoriya M, Kaul KK (2008). Effect of tobacco chewing by mothers on placental morphology. Indian Pediatr, 1983.

Ashfaq M, Channa MA, Malik MA, Khan D (2008). Morphological changes in human placenta of wet snuff users. J Ayub Med Coll Abbottabad, 2008.

Bhisey RA (2012). Chemistry and toxicology of smokeless tobacco. Indian J Cancer, 2012, doi:10.4103/0019509X.107735.

Boffetta P, Hecht S, Gray N, Gupta P, Straif K (2008). Smokeless tobacco and cancer. Lancet Oncol, 2008, doi:10.1016/ S1470-2045(08)70173-6.

Borhade A (2011). Migrants' (Denied) Access to Health Care in India. Intern Migr India Initiat Natl Work Intern Migr Hum Dev India 6-7 December 2011 Indian Counc Soc Sci Res (ICSSR), New Delhi, India. 2011.

Caleyachetty R, Tait CA, Kengne AP, et al (2014). Tobacco use in pregnant women: Analysis of data from Demographic and Health Surveys from 54 low-income and middle-income countries. Lancet Glob Heal, 2014, doi:10.1016/S2214109X(14)70283-9.

CDC Foundation, World Health Organization, Lung Foundation. The GATS Atlas Global Adult Tobacco Survey [Internet]. 2015. Available from: http://gatsatlas.org/downloads/GATSwhole-book-12.pdf, accessed on January 11, 2020.

International Institute for Population Sciences (IIPS) and ICF. National Family Health Survey (NFHS-4), 2015-16: India. Mumbai; 2017.

Jatoi I, Cummings KM, Cazap E (2009). Global tobacco problem getting worse, not better. J Oncol Pract, 2009, doi:10.1200/ jop.0918501.

Mughal F, Memon ZA, Qureshi SS (2019). Effects of use of smokeless tobacco on microstructure of human placenta. J Soc Obstet Gynaecol Pakistan, 9.

National Tobacco Control Cell, Welfare, Ministry of Health and Family Welfare, Government of India. Operational Guidelines: National Tobacco Control Programme [Internet]. 2015. Available from: https://nhm.gov.in/NTCP/ Manuals_Guidelines/Operational_Guidelines-NTCP.pdf, accessed on January 12, 2020.

Pratinidhi A, Gandham S, Shrotri A, Patil A, Pardeshi S (2010). Use of "Mishri" A smokeless form of tobacco during pregnancy and its perinatal outcome. Indian J Community Med, 2010, doi:10.4103/0970-0218.62547.

Rogers JM, Tobacco and pregnancy. Reprod Toxicol, 2009.

Roth MA, Aitsi-Selmi A, Wardle H, Mindell J (2009). Underreporting of tobacco use among Bangladeshi women in England. J Public Health, https://doi.org/10.1093/pubmed/ fdp060.

Shipton D, Tappin DM, Vadiveloo T, et al (2009). Reliability of self reported smoking status by pregnant women for estimating smoking prevalence: A retrospective, cross sectional study. BMJ (Online), https://doi.org/10.1136/ bmj.b4347.

Suliankatchi RA, Sinha DN (2016). The human cost of tobacco chewing among pregnant women in India: A Systematic Review and Meta-analysis. J Obstet Gynecol India, 2016.
Tata Institute of Social Sciences and Ministry of Health and Family Welfare Government of India. Global Adult Tobacco Survey GATS 2 India 2016-2017 Report. Report. 2017. Available from: http://cancerindia.org.in/wp-content/ uploads/2018/09/GATS_2_India-Report.pdf, accessed on December 19, 2019.

Yadav A, Singh PK, Yadav N, et al (2020). Smokeless tobacco control in India: Policy review and lessons for high-burden countries. BMJ Global Health, 2020. doi:10.1016/j. reprotox.2009.03.012.

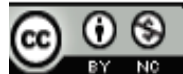

This work is licensed under a Creative Commons AttributionNon Commercial 4.0 International License. 